\title{
Translation Briefs on the Epic Gesar and Its Signicance
}

\author{
Xueyun Zang \\ School of Foreign Languages \\ Qilu Normal University \\ Jinan, China
}

\begin{abstract}
Abstrcat-This paper focuses on the summary of the history of translation of Tibetan Epic Gesar. We can divide the translation versions into the following: Tibetan to Chinese, Tibetant to other minor ethic languanges, Tibetan to foreing languages except English, Tibetan to English. In the paper, the author describes the outlines translation version of Gesar and points out the significant of translation Gesar into other languages.
\end{abstract}

Keywords-Gesar; translation; significance

\section{INTRODUCTION}

Gesar since created was not only popular in the Tibet area of our country, affecting the indigenous Tibetan people, but also spread to the surrounding countries and regions that are adjacent to the Tibetan, forming the Gesar with local and national characteristics.

In this process, translation is the direct carrier. It can be said that without translation, there is no culture exchange between all ethnic groups and countries nor the widely spread of Gesar in various countries and regions of the world.

Gesar and its translation research, generally includes four kinds of circumstances:

- Been translated into other ethnic characters ;

- Been translated into Chinese ;

- Been translated into foreign languages ;

- By other minority words translated into Chinese or foreign, or by foreign translated into Chinese, Mongolian, Tibetan, etc.

To conduct academic research, at the same time, in order to clarify the translation of Gesar development, we summarize the four circumstances raised by the professor tashi eastern pearl into people of the translation, Chinese translation and translation. People of the translation means Gesar is translated into other domestic ethnic language. Of course, it includes nations that have no word such as the monguor people. Chinese translation, namely, Gesar is translated into Chinese. Chinese, in fact, has become the

This paper is sponsored by Shan Dong Provincial Social Science union Program: Research on the Translation and Introduction of Gesar in Europe and America Research Number: 16CWZJ04) common language of all fraternal nations in China. No matter now or in the future, in our country, The people who know Tibetan are always few and the vast majority of readers will understand and appreciate the Gesar by Chinese translation. The Gesar may be translated into foreign language by translated into Chinese first in the future. At least, Chinese translation can be referred. Thus it can be seen that the Chinese translation of Gesar is of great importance in the translation. As a matter of fact, so far, the Tibetan edition of Gesar has achieved the biggest achievement in the process of Chinese translation. Outside, refers to Gesar translated into language of other countries in the world, such as French, German, Russian, English, etc. According to incomplete statistics, now the Gesar has spread all over the world, which are translated into French, German, Russian, English, Indian, Latin, Liepu, Bruce shaath Motofumi, Turkey, Japanese and more than 10 languages. Although the original Tibetan is given priority to rhyme, scattered rhyme combinition form far and is mainly the compilation of prosify, different versions of the Gesar have played an important role in cultural transmission. At least to the target language readers introduced the Tibetan heroic epic, make the target language readers understand the tibetans in China has a great hero epic, as well as Tibetan studies provides a blueprint from country to country.

\section{GeSAR'S PEOPLE OF THE TRANSLATION}

Tibetan is one of a minority that has both language and word among 55 minorities in our country. In addition, according to statistics, Tibetan information stored in our country is the most populous ethnic languages in addition to the Chinese data. As a Tibetan oral literature "Gesar" in the beginning of creation, was also a Tibetan singing. It gradually formed the hand-written, engraved forms and later the printed copy. At the beginning of Gesar's formation, during the process of spreading to other ethnic groups that lived together with Tibeter and ethnics around, there's no doubt that interpretation as a mundliterature had played an important role. Or interpretation which appeared first formed the translation of other nation's words gradually. The role of interpretation is irreplaceble, especially in the process of spreading to the ethnic groups without character, such as Monguor people. 
Gesar as a living epic, in addition to spreading in the Tibetan masses also circulated widely in the domestic Mongolian, tu, yugur, pumi, salar, lisu, naxi, bai and so on.

\section{Chinese Translation OF GESAR}

According to the available information, it is renowned tibetologist Mr Ren who translated Gesar into Chinese in our country. $1928 \sim 1929$, Mr. Ren visited Kangba alone, witnessed the Tibetan people around together to listen to Gesar by rapper for several days and he was so shocked by that. Later neosaurus county ji mo zhuoma read this sichuan RaoLu village club (a hidden Gesar of the department, and Invited Tong si translated and record it.After backing to Chengdu, he corrected and arranged translation with his wife. The passage was translated into the current classical and vernacular style, which was divided into two and published in the Sichuan daily supplement titled "hidden in The Three Kingdoms" and "hidden in The Three Kingdoms" in Dcember 1930. Later they merged into one.Though the translation is only more than one thousand five hundred words, but it is not only the earliest Chinese translation of Tibetan Gesar, but also the symbol of scholars in our country for the first time to introduce the Gesar to the academic circles and mainland readers, and academia to Tibetan epic Gesar. It represents the beginning that researchers who don't belong to Tibetanos study Gesar and opened the prologue of Gesar's Chinese translation. Mr. Ren, as it were, for the first time Introduced the great epic to Chinese readers, opened the first Chinese translation of Gesar, so he did a great contribution to it.

In 50 s and $60 \mathrm{~s}$, the collecting and organizing work of Gesar was carried out fully, which was followed by the translation and research of epic and started the first climax of domestic research in the history of character. Northwest university for nationalities is the first to start collecting and organizing Gesar after the founding of The People's Republic of China. Yu Xizhe teacher firstly went to Tibet, Qinghai, Sichuan, Gansu, Tibetan to collected and organized Gesar. They have collected the SangLing wars, the ridge and zhu ancient wars such as this, but due to the change of the social situation at that time, the work was suspended. During this period, federation of Qinghai province played an important role. From 1957 to 1962, professor Wang xiyuan, Cai dan xia rong and Gong que cai dan from northwest university for nationalities all served to participate in the work of collection and translation about Gesar. The journal of Qinghai Lake also became the main propaganda of Gesar. In 1959-1964, qinghai federation organized the translator translated the Tibetan Gesar this division of 28 more than 50 copies, your chapter points in this one. Qinghai lake in 1957 of phase 7 published brief play of South the pursuit of Gesar which was telled by Huajia and translated by Jinfang.It also published "hall invasion" in 1959, 10-11 and "department of horse racing king" in 1960, 5-8, which caused quite a reaction. In May 1962 the Shanghai literature and art publishing house published the war of king Gesar, HuoLing (I), which attracted the attention of both at home and abroad and had a favorable review in readers.
Professor Wang xiyuan, from northwest university in Gansu province made a great contribution. Mr Wang took part in the translation and research of Gesar since 1957. Since 1978, especially after 1982, as the group leader of Gesar translation in northwest institute for nationalities, he specialized in the translation and research of Gesar and then achieved fruitful results. Mr. Wang's contribution also lies in he put forward the conception of the points and division. $\mathrm{Mr}$ wang's translation has its unique style and characteristics and his translation reserves the feature of folk oral literature. Its content is loyal to the original and the language is plain, which is easy to read.We can say that Mr Wang has made a great contribution to the spread of this epic's translation, so he is known as "Gesar study leading" and "Gesar, learn the blazers" by scholars.

\section{GeSAR OUTSIDE TRANSLATION}

According to statistics, Gesar has been published abroad in Mongolian, Russian, English, French, German, Hindi, Urdu, Spanish, Japanese and more than 20 languages. Research indicates that this chapter has been published by more than 150 kinds of different departments and more than 300 books. Objectively speaking, it is because of the early translation of the epic that the world has a chance to contact and understand the magnum opus "encyclopedia" of ancient Tibetan culture; at the same time,because of different translation of various periods in history, epic eventually come to the attention of relevant scholars in the world. With regard to the study of Gesar, scholars whether from the text, language, culture or from the ethnology, religion, folkways, anthropology, folklore and many other different and endless perspectives make it become a cosmopolitan dominant discipline. This part involves larger time span and more complex number, so it is more representative and has larger influence on the translation.

Outside, Gesar was vigorous in history, so to speak. Its foreign translation promotes the spread of Tibetan culture in the foreign and makes foreign public have a chance to understand the world's longest epic. However, we should also see the worries behind the hustle and bustle. That is to say, so far, the foreign translation of Gesar is essentially compilation and translation. To some extent, what can accurately reflect the truth of Tibetan culture are the contemporary scholars and researchers about mining.

\section{TRANSLATION OF GESAR}

Roughly went through several different stages:

- In Gesar, above all outside translation sections, French female scholar, D.Neil went to Tibetan areas in our country in the 1930s. With the help of Yong Deng lama, she translated the Ridge Siperman King Gesar by listening to singing artist and consulting Gesar woodblock prints. Two years later, in 1933,Waller•ShuDeNi translated it into English and then published in London. After that, it was reprinted in 1959. In 1979, the English version of the book was published in New York. What I get right now are the reprint of Shambhala Boston and London edition, 
which were published in 1987. This version also points that the chapter of Gesar is shallow interpretation and description on the whole and it must have been the Frenchman's reading interest.At the same time, they are interested in the east, which is associated with a mysterious world of adventure. Although the author did a lot of rewriting and some basic rhyme in prose translation, it become one of the earliest Gesar study version for western scholars.

- In the process of translation, Gesar can be called a rising star in the United States. Several consecutive English translations has been published in the United States, which has a close relationship to the the spread and development of Tibetan Buddhism in North America after world war II .In 1927, Ada·Zett Lin Yi meng announced it as reference and English translation of gus, Gesar Khan A Legend of Tibet was published by George dolan press in New York. In 1911 , the plan of congress library launched the "Tibetan art and culture series" Gesar! The Wondrous Adventures of King Gesar, one of the English dharma of California press. The language of translation was fluent and it reshaped King Gesar in contemporary English story. As a popular book, it was welcomed by the readers. Douglas Pan Nick's The Warrior Song of King Gesar was published from Boston wisdom press. As a translator, his translation may be arbitrary but the content is easy to understand. It is convenient for readers who have no background of Tibetan studies and expand the influence in the contemporary American readers.

- Gesar of translation in China starts late, but due to the unique advantages__ "Gesar in China", the translation of epic also achieved some results. The domestic Gesar worth nothing because generally it is based on the Chinese translation. In 1987, JiangBianJiaCuo cooperated with WuWei had published three volumes Gesar translation and the Historical complete biography. This is a collection of literariness and academic writing. And for the first time, this epic is introduced to han Chinese readers in the relatively complete form. In 1989, It won the outstanding book award by the general administration of press and publication and it was reprinted and revised by the writers press in 1997. In 2006, China intercontinental pressed the historical complete biography on part II .Liaoning publishing house and Wuzhou press worked together to streamline the King Gesar. The traditional style with this form, scattered rhyme and illustrated are a total of 12 chapters. WangGuozhen, ZhuYongmei cooperated in 2009, with JiangBianJiaCuo and WuWei jointly published Published the Historical complete biography as dibon by compiling. It was published jointly by Liaoning press and Wuzhou press, which originally only control the Chinese and English translation of Gesar. With chapter points for the translation of the form, rhyme combination, narrative, and illustrated, Gesar has become a landmark in domestic English translation.

Retrospect the history of Gesar translation, we can tease out an idea like this: In the early 20th century, accompanied by the missionaries in China, along with the embryonic form of overseas Sinology, all eyes turned to Chinese traditional culture. These foreign promotion of culture also caused more and more people come to China to travel and adventure. The English version of Frank's Ladakh's next version and the English translation of Viniar's Ridge Superman King Gesar is produced in this period. It also represents the center of overseas sinology in Europe at that time. With the end of world war II, the rise of global overseas sinology has been to North America.After a period of time, the introduction of three different translations of Gesar appeared in the United States.This was related with the development and and spread of the "Chinese hot" in Chinese classics. In the big background of improving the power of Chinese soft culture and "going out", the domestic academic circles have carried on the contemporary academic thinking, represented by Zhou guozhen's English translation of Gesar, which took a solid step.

\section{REFERENCE}

[1] Zhaxi Dongzhu. History of Gesarology[M]. Lanzhou: Gansu Ethnic Press, 2002:113.

[2] Jiangbian Jiacuo. Exploration on Gesar[M]. Xining: Qinhai Renmin Press, 1986:340.

[3] Zhaxi Dongzhu. On the Translation Questions of Gesar[J]. China Translation, 2004.2:87. 